\title{
«Texte et paratexte dans « Nulle part dans la maison de mon père " d'Assia Djebar. "
}

\section{Ibrahim Abouelmaati Ibrahim Elmorsy* \\ dr.ibrahimmaati@gmail.com}

\section{Résumé :}

Assia Djebar est l'une des écrivaines algériennes d'expression française la plus distinguée. Elue à l'Académie française en 2005, Assia Djebar est l'auteure de quinze romans abordant une part très importante de l'Histoire de son pays, et défendant surtout la liberté de la femme arabe. Son œuvre romanesque a été traduite en plusieurs langues étrangères.

Parmi son œuvre romanesque très touffue, nous avons opté pour son dernier roman: "Nulle part dans la maison de mon père », édité pour la première fois en 2007. La problématique de notre recherche porte comme titre : " Texte et paratexte dans « Nulle part dans la maison de mon père » d'Assia Djebar." Ce roman contient pas mal d'éléments paratextuels qui nous étonnent et qui nous poussent à traiter ce sujet. Nous avons analysé la relation étroite entre le contenu du roman et les éléments paratextuels qui regroupent le nom de l'auteur, le titre de l'ouvrage, la maison d'édition et son label, l'illustration spécifique, la dédicace, les intertitres, l'épilogue, la postface et la quatrième couverture.

Djebar présente un roman tout à fait différent des autres par sa structure singulière, elle a réussi à bien valoriser chaque élément paratextuel du roman. Cet ouvrage représente une marque distinguée dans son parcours romanesque, elle a excellé à toucher son lecteur à partir du titre du roman.

Mots clés : Texte - paratexte - titre - nom de l'auteur- indication générique- maison d'édition- page de titre- dédicace- épigraphe intertitres- épilogue- postface- quatrième de couverture.

\footnotetext{
Maître de conférences à la Faculté des Lettres, Université de Kafrelsheikh.
}

(Texte et paratexte dans ...) Ibrahim Abouelmaati Ibrahim Elmorsy 
Assia Djebar est l'une des écrivaines algériennes d'expression française les plus nommées et les plus intéressées aux problèmes de son pays d'origine en général et à la situation de la femme arabe en particulier. Née le 30 juin 1936 dans un petit village algérien et morte en 2015, Assia Djebar a réalisé une renommée considérable comme écrivaine et historienne. Membre de l'Académie française de 2005 jusqu'à sa mort, cette écrivaine, l'une des plus influentes de sa génération, occupe une place à part dans la littérature de langue française pour la totalité de son œuvre littéraire. Elle a réussi à s'imposer; c'est bien sûr grâce à ses contributions littéraires couronnées de succès. Trudy AgarMendousse apprécie que :

"L'œuvre d'Assia Djebar occupe un rang de $1^{\text {er }}$ ordre dans la littérature algérienne grâce au fait que Djebar a été la première femme à prendre la plume pour écrire sur la femme et son corps (...)tout au long de son ouvre Djebar s'interroge sur l'identité des femmes d'Algérie et sur sa propre identité de femme franco algérienne; sur le corps féminin; sur son rôle d'écrivaine et sur la violence qui a déchiré son pays. » ${ }^{(1)}$

Nous avons choisi un roman remarquable dans sa fresque romanesque : "Nulle part dans la maison de mon père », édité pour la première fois en 2007 aux éditions Fayard, puis réédité aux éditions Sedia, Acte sud et Babel ; un roman qui se distingue des autres par sa structure particulière. Ce roman contenant 451 pages se divise en trois grandes parties, presque équilibrées en volume, suivies d'un épilogue et une postface, en l'absence d'une introduction. "Nulle part dans la maison de mon père » contient pas mal d'éléments paratextuels qui nous étonnent et qui nous poussent à choisir ce sujet. Le roman est bien structuré comme si l'auteure présente une thèse de doctorat et pas un ouvrage romanesque; ce qui nous attire l'attention, c'est notamment le titre du roman et tout ce qui l'accompagne; puis viennent les

(Texte et paratexte dans ...) Ibrahim Abouelmaati Ibrahim Elmorsy 
autres données paratextuelles dont chacune suscite notre curiosité et nous pousse à chercher dans le roman la réponse de quelques questions comme : Est-ce qu'il y a une relation qui les lie ensemble ou non? Est-ce que ces éléments se rattachent étroitement au texte ? Est-ce que leur objectif est d'éclairer le roman avant la lecture ? Est-ce que ces données paratextuelles portent un message implicite ou explicite? Est-ce que ces éléments utilisés par Assia Djebar se différencient des autres ?

Le dernier roman d'Assia Djebar est accompagné d'un certain nombre d'éléments paratextuels intéressants qui dévoilent l'identité de cet ouvrage avant même de commencer à lire. Ces éléments entourant et pénétrant le texte jouent un rôle primordial chez le lecteur; ceux-là deviennent un outil indispensable soit pour l'écrivain, afin de présenter son livre, soit pour le lecteur, afin de bien déterminer pour quelle raison il va acheter le livre. C'est pourquoi la problématique de notre recherche porte comme titre: " Texte et paratexte dans "Nulle part dans la maison de mon père » d'Assia Djebar."

Gérard Genette donne une définition claire du terme " le paratexte": "Le paratexte est donc pour nous ce par quoi un texte se fait livre et se propose comme tel à ses lecteurs, et plus généralement au public. Plus que d'une limite ou d'une frontière, il s'agit ici d'un seuil ou [...] d'un "vestibule » qui offre à tout un chacun la possibilité d'entrer ou de rebrousser chemin ». ${ }^{(2)}$

D'après Gérard Genette, le paratexte se divise en deux parties essentielles : le péritexte qui regroupe tout ce qui se situe autour du texte comme le titre, le sous-titre, la page de titre, la dédicace, l'épigraphe, les intertitres, ...etc. et l'épitexte contient l'extérieur $\mathrm{du}$ livre comme les interviews, les entretiens, les correspondances...etc. $^{(3)}$; ces deux éléments se partagent le champ spatial du paratexte.

(Texte et paratexte dans ...) Ibrahim Abouelmaati Ibrahim Elmorsy 
Alors, il est à noter que notre analyse du roman étudié sera basée sur le péritexte, en l'absence de l'épitexte. L'auteure a éliminé cette deuxième catégorie du paratexte ou peut-être n'a pas voulu la présenter dans le roman à étudier, c'est pour bien souligner à son lecteur que "Nulle part dans la maison de mon père" va terminer sa création romanesque, celui-là n'a pas besoin d'autres éléments extérieurs pour aider le lecteur à bien découvrir le roman; aucun élément de l'épitexte, bien sûr publié avant la parution du roman, n'ajoute rien d'important à cet ouvrage romanesque volumineux. Ainsi, Assia Djebar cherche à présenter un texte tout à fait original, unique en son genre.

Commençons par "la page 1 de couverture"(•) qui représente un élément essentiel du "péritexte éditorial ${ }^{\text {(*), }}$, celle-là regroupe de nombreux éléments dont les plus importants sont le nom de l'auteur, le titre de l'ouvrage, le nom de la maison d'édition et son label, et l'illustration spécifique.

\section{Le nom de l'auteur:}

Il est à noter que le nom Assia Djebar est un cas particulier pour le roman objet d'étude par rapport à ses autres romans; cela revient en premier lieu à l'importance donnée au nom de l'auteur comme élément paratextuel méritant d'être analysé; et en second lieu à la relation étroite entre la vie privée de l'auteure et les événements du roman.

Le nom de l'auteur vient en haut de la première couverture en lettres toutes majuscules : ASSIA DJEBAR qui est le pseudonyme de l'auteure; d'après Genette, ici l'auteur "signe d'un faux nom, emprunté ou inventé : c'est le pseudonymat." ${ }^{(4)}$; le vrai nom de l'auteure du roman est tout-à-fait loin de cette dénomination, elle a voulu cacher sa véritable identité, elle avait peur de sa famille, et surtout de son père. L'auteure est issue d'une

(Texte et paratexte dans ...) Ibrahim Abouelmaati Ibrahim Elmorsy 
famille arabe musulmane, une famille qui garde les traditions des ancêtres ; et cela apparaît clairement dans le vrai nom de l'auteure: Fatima-Zohra, c'est un nom compliqué, complètement relatif à la religion adoptée par sa famille : l'islam.

Lors d'une interview, Djebar a indiqué les raisons pour lesquelles elle a choisi de prendre un pseudonyme lors de la parution de son premier roman "La Soif" en 1957 :

"Ma seule crainte à l'époque était que mon père tombe sur ce roman (la soif) un peu osé. Il me fallait un nom de plume, on m'a proposé Assia, qui est celle qui console en arabe, et Djebar; qui veut dire "intransigeante". Ce nom m'a plu car je savais que pour faire ouvre littéraire il fallait être intransigeante, avec soi même bien sûr!» (5)

Assia Djebar explique pour la deuxième fois comment elle a opté pour ce pseudonyme :

" À vingt ans, le jour où j'ai signé mon contrat, il me fallait un pseudonyme. (...) Je ne voulais pas que mon père et ma mère sachent que j'ai écrit un roman. J'allais chez l'éditeur. A ce moment-là, (...) je vais prendre Djebar. J'ai pris le nom d'Assia parce que je le trouvais assez beau. Plus tard quelqu'un m'a dit: "Assia, c'est la fleur immortelle»." (6)

Assia est un prénom très répandu chez les musulmans ; mais il prend deux formes d'orthographe assez proches : Assia et Asiya: "Pour les musulmans, Asiya fut l'épouse du Pharaon qui recueillit Moïse. Elle accède au rang de femme parfaite."(7), Quant au prénom Djebar, il s'utilise rarement et concerne seulement les garçons. Ce prénom à double sens réfère premièrement à l'un des noms d'Allah "Al-jabar", et deuxièmement à la personne forte pleine de rigueur. Mounira Chatti estime que : "Assia, c'est la consolation, et Djebar, l'intransigeance. "(8)

Assia Djebar a choisi un pseudonyme tout-à-fait arabe pour exprimer son attachement étroit à sa société d'origine et à son

(Texte et paratexte dans ...) Ibrahim Abouelmaati Ibrahim Elmorsy 
héritage arabo-musulman, malgré sa passion pour la langue française, la langue de l'Autre :

"Elle est, par son père instituteur, une fillette algérienne élevée dans l'amour de la langue française, un pur produit de la méritocratie coloniale et républicaine. Mais elle est aussi par sa mère, une berbère riche de son héritage arabo andalou, qui vit au cour des assemblées de femmes, au rythme des mariages des circoncisions ou des après-midis au hammam." ${ }^{\prime(9)}$

\section{Le titre du roman :}

Le titre est toujours et à jamais le premier élément paratextuel qui attire et éveille l'attention du lecteur; pour cette raison, il occupe la place primordiale de toute analyse pour sa liaison forte au contexte du roman. Charles Grivel le considère comme un "signe par lequel le livre s'ouvre: la question romanesque se trouve dès lors posée, l'horizon de lecture désigné, la réponse promise." ${ }^{(10)}$, Léo Hoek affirme l'importance incontestable de l'analyse du titre : "Il faut commencer l'étude du texte par celle de son titre.» ${ }^{(11)}$.

Pour son dernier roman, Assia Djebar donne un titre à la fois bizarre et attrayant qui contient le pour et le contre en même temps, un titre personnel à travers l'emploi de l'adjectif possessif "mon", un titre symbolique qui exprime un sentiment de malaise, un titre paternel qui s'attache étroitement au personnage du père ; Ridha Bourkhis apprécie que :" La narratrice donne la maison et dans le même temps le vide." ${ }^{(12)}$. Le titre du roman vient en lettres majuscules et en haut de la première couverture au-dessus du nom de l'auteure : "NULLE PART DANS LA MAISON DE MON PÈRE". On est tout-à-fait d'accord avec Vincent Jouve qui distingue entre "des titres qui accrochent et des titres qui rebutent, des titres qui surprennent et des titres qui choquent, des titres qui enchantent et des titres qui agacent" ${ }^{\prime \prime 213)}$

(Texte et paratexte dans ...) Ibrahim Abouelmaati Ibrahim Elmorsy 
En feuilletant le roman, on constate que le titre s'attache étroitement au contenu du texte, et surtout à l'état d'âme de l'auteure ; alors ses fonctions sont thématiques, selon Gérard Genette $^{(14)}$; mais d'après Léo H. Hoek, c'est un titre subjectival qui sert à désigner le sujet du texte. ${ }^{(15)}$ On est d'accord avec Genette dans son point de vue concernant l'ambiguïté du titre dans certains cas :" la relation thématique peut être ambiguë, et ouverte à l'interprétation." ${ }^{(16)}$; une étroite relation lie le titre du roman "Nulle part dans la maison de mon père" et la plupart des éléments paratextuels (contextuels), surtout les intertitres du roman: on constate l'intérêt donné par l'auteure à la première partie qui contient des intertitres liés étroitement au sens global du titre: " La jeune mère, Les larmes, Le tout premier livre, Le père et les autres, La bicyclette, le jour du hammam, le petit frère, Le petit frère, La chambre paternelle"; on trouve également dans la deuxième partie:" Le piano, Corps mobile, Le monde de la grandmère maternelle, L'été des aïeules"; et enfin l'auteure retourne de nouveau au titre du roman à travers la troisième partie: " Encore au village, Lettre déchirée, La famille à Alger, Ce matin-là"; même l'épilogue n'était pas à l'écart du titre du roman :" Le silence ou les années-tombeaux, La jeune fille sauvage". L'intertitre de la postface "Silence sur soie" ou "l'écriture en fuite" se lie aussi au titre du roman. ${ }^{(\boldsymbol{(})}$ Assia Djebar a bien présenté les données paratextuelles tout au long du roman, celleslà se distinguent des autres par son abondance, sa distribution bien équilibrée et aussi leur rôle à esquisser une part réelle de la vie de l'auteure.

En essayant de trouver l'objectif essentiel pour lequel est apparue cette dénomination du roman, nous devons nous plonger un peu dans l'autobiographie de l'auteure, et plus précisément dans sa relation paternelle. Il est à noter que "Nulle part dans la maison de mon père" peut être " le roman le plus explicitement

(Texte et paratexte dans ...) Ibrahim Abouelmaati Ibrahim Elmorsy 
autobiographique d'Assia Djebar"(17); cet ouvrage romanesque raconte beaucoup de détails importants concernant la vie de l'auteure depuis ses premières années jusqu'à son entrée à l'école normale supérieure de Paris; période intéressante pleine d'événements attractifs et impressionnants dans l'itinéraire familial de l'écrivaine.

À travers le titre, le lecteur comprend vite qu'il y a une grande distance entre l'héroïne et son père, et qu'il n'y a pas de place pour la narratrice dans la maison de son père; mais ce titre nous amène à chercher son vrai sens, en essayant de dévoiler quelle relation entre le père et la fille narratrice. "Nulle part" s'écrit toujours comme ça, nulle est le féminin de nul, cet adjectif s'accorde ici en genre avec "part". "Nulle part" qui signifie "d'aucun côté, en aucun endroit ${ }^{\text {"(18) }}$, est la négation de "partout" et dans la plupart du temps est précédé par "ne"; ici Djebar l'utilise au début de son titre du roman comme si quelqu'un l'interroge: tu vis où? Et elle répond "Nulle part"; mais elle l'a suivi de "dans la maison de mon père". Ridha Bourkhis apprécie que : "l'ombre du père était encore trop imposante pour que la narratrice s'en libérer des dizaines d'années plus tard."(19) Assia Djebar a voulu exprimer sa peur de son père dont la maison est basée sur les traditions et les coutumes des ancêtres. Et cela apparaît nettement dès le début du roman où la narratrice nous raconte son allée avec la mère, alors qu'elle a encore trois ans, pour rendre visite familiale ; là-bas, elle montre sa différenciation des siens en disant :" Je me sens la fille de mon père". Une forme d'exclusion-ou une grâce? "NMP. ${ }^{(*)}$ $p .19$, cette phrase, exprimant le contentement et la fierté d'être la fille de cet instituteur ou peut-être l'ironie et le mécontentement d'être assujettie à des règles imposées par le père, est suivie d'une interrogation qui éclaircit et interprète l'intention de l'auteure ; dans cette période de sa vie, elle n'était pas capable de percevoir la vérité; mais dans le même contexte, elle exprime franchement

(Texte et paratexte dans ...) Ibrahim Abouelmaati Ibrahim Elmorsy 
sa relation maternelle; en parlant de sa mère, elle dit :" je me sens si fière de paraître à ses côtés. "NMP., p.15. En ce qui concerne le roman, Ridha Bourkhis apprécie que:

"Installé dans un caractère, toujours en surplomb de la mère, le livre lui est un hommage (...) il est à lire en regard de l'héritière de ce père, dans la reconnaissance d'une filiation physiologique, mais surtout spirituelle, via les qualités qui lui sont rapportées, et jusque dans l'acceptation de ses intransigeances." (20)

Ce n'est pas seulement le titre du roman qui insiste sur la présence d'une relation un peu compliquée et même ambiguë entre la narratrice et le père, mais aussi la description présentée ; la petite fille narratrice dit qu':" Il ne rit pas, le père. Il constate tout haut, presque pour lui seul. D'ordinaire, il ne parle pas à table. Quelque chose de sa sévérité d'instituteur subsiste chaque soir dans la cuisine où l'on mange en silence... "NMP., p.48.

Dans la plupart de son œuvre romanesque, Djebar donne toujours une place considérable à la souffrance des femmes et leur sujétion devant leurs époux; ici, la narratrice exprime à quel point arrive la relation entre ses parents : " il n'y avait pas entre eux vraiment une connivence, mais elle ne protestait pas... "NMP., p.56

La première occurrence du titre du roman paraît assez tard dans la trame narrative, la narratrice parle de l'une de ses condisciples de l'internat ; c'est Mounira qu'elle considère comme une fausse camarade ; celle-ci cherche aussi la liberté loin du domicile paternel. La narratrice l'entend soupirer à mi-voix :" Nulle part, hélas, nulle part dans la maison de mon père." NMP., p. 223; ainsi la narratrice souligne la souffrance dans laquelle vit une génération tout entière.

Le titre se répète une autre fois à la fin de la troisième partie du roman, la narratrice s'adresse à elle-même en recherchant sa place,

(Texte et paratexte dans ...) Ibrahim Abouelmaati Ibrahim Elmorsy 
et en se demandant si elle est la fille qui déclare "soudain presque à la face du monde : "Nulle part dans la maison de mon père"?" NMP., p.404

Dans l'épilogue, on retrouve le titre pour la troisième fois répété, mais la narratrice pose à nouveau une question concernant toute sa génération des jeunes filles :" pourquoi, mais pourquoi faut-il que je me retrouve, moi et toutes les autres, "nulle part dans la maison de mon père"?" NMP., p.433; la narratrice poursuit sa perte de lieu dans la maison de son père, même après sa mort. Assia Djebar critique ici l'injustice de la société maghrébine dite musulmane où les frères privent leurs sœurs de leurs droits dans l'héritage; c'est peut-être la dernière justification du titre qui exprime la quasi-absence totale des droits des femmes parmi les hommes :" Je n'ai plus de "maison de mon père". Je suis sans lieu, là-bas, non point seulement parce que le père est mort, affaibli, dans un pays dit libéré où toutes les filles sont impunément déshéritées par les fils de leurs pères." NMP., p.427

Passons à un autre élément paratextuel éditorial très important et très significatif dans cet ouvrage, c'est l'indication générique "Roman", qui représente, d'après Genette, une "annexe du titre, plus ou moins facultative ${ }^{\prime \prime(21)}$, et que l'on retrouve à la fois sur la page 1 de couverture et sur la page de titre en face de "DU MÊME AUTEUR" dont la fonction est de faire connaître au lecteur les titres des autres œuvres de l'auteur; en bref son catalogue personnel. ${ }^{(22)}$ D'après Genette, l'emplacement normal de l'indication générique est, soit la couverture, soit la page de titre, soit les deux.

A travers cet indice paratextuel, l'auteure a voulu informer son lecteur dès la première vue le genre de son ouvrage en insistant, par la répétition du terme, sur la part littéraire et le caractère fictif; peut-être elle a également désiré prouver son habileté à mélanger

(Texte et paratexte dans ...) Ibrahim Abouelmaati Ibrahim Elmorsy 
le réel et la fiction dans le même ouvrage. Mounira Chatti donne une définition de la littérature en disant : "La littérature est le lieu où se déploie la liberté, celle de déconstruire le monde, celle d'affirmer un engagement poétique et politique." (23)

On va maintenant au quatrième élément paratextuel éditorial trouvé sur la couverture: c'est la maison d'édition qui joue un rôle très important dans la diffusion de l'œuvre et sa distribution; elle représente le pont qui relie entre deux éléments: l'auteur et le lecteur; c'est un élément paratextuel indispensable dans toute œuvre littéraire. Sur la première couverture du roman, on lit BABEL et on trouve aussi son label dessiné en haut de la page à droite, c'est une collection d'édition de livre au format poche, créée par les éditions Actes Sud en 1989.

L'illustration spécifique trouvée dans la première page de couverture du roman est une image à la fois très simple et très profonde qui représente également un élément contextuel pour son attachement étroit aux événements du roman; elle contient des éléments relatifs au littoral algérien. Cette image souligne l'attachement de l'auteure à son ancienne ville "Césarée" située sur la côte méditerranéenne de l'Algérie. C'est pour cela, on trouve une muraille haute d'une maison donnant sur une autre maison basse. À côté des maisons, il y a deux arbres qui indiquent le calme et la simplicité du paysage rural. Au voisinage, il y a également une mosquée située près la mer où se trouvent des bateaux. Tous ces éléments soulignent bien le milieu dans lequel a vécu l'écrivaine. Cette image exprime aussi la passion de l'écrivaine pour ces paysages absents à ce moment-là de sa vue, surtout la maison paternelle au village.

La mosquée, lieu de culte pour les musulmans, apparaît avec ses deux dômes en soulignant la religion à laquelle convertit la famille d'Assia Djebar. À travers cette image, l'écrivaine exprime

(Texte et paratexte dans ...) Ibrahim Abouelmaati Ibrahim Elmorsy 
son originalité et sa loyauté; malgré sa notoriété brillante, elle ne rompt pas avec ses racines profondes.

Ainsi, il est remarquable que notre écrivaine a une grande prédilection pour la mer, le village, et la maison paternelle, toujours présents dans le roman. C'est pour cette raison qu'elle a donné à la troisième partie du roman un titre tout à fait attaché à la mer " Celle qui court jusqu'à la mer"; dans cette partie on trouve une section titrée "Promenades au port ", et une autre intitulée " Encore au village "; et bien sûr le titre du roman analysé est le bon témoignage de sa recherche continue de trouver un lieu dans la maison de son père.

Assia Djebar a excellé dans son choix de l'image de la première couverture, une photographie qui éveille aussi la curiosité du lecteur qui cherche toujours à travers les événements du roman une justification adéquate à tous ces éléments dessinés.

\section{La dédicace:}

Autre élément paratextuel très simple dans la plupart des œuvres littéraires, c'est la dédicace qui exprime une certaine relation entre l'auteur et la personne à laquelle est dédiée l'œuvre. Ce sont toujours des personnes proches de l'auteur, soit par un lien de sang, soit par une relation d'amitié, soit par une autre relation inconnue et propre seulement à l'auteur. Dans le roman à étudier, Assia Djebar a dédié son œuvre à une personne réelle, plus ou moins connue, c'est Gayatri Chakravorty Spivak qui est à la fois écrivaine, philosophe, professeur d'université, traductrice, professeure, et critique littéraire. Gayatri est américaine d'origine indienne, intéressante à la littérature et l'Histoire postcoloniale. Il est à noter que cette théoricienne s'attache fortement à l'œuvre d'Assia Djebar comme a affirmé Philippe Daros, Professeur à l'Université Sorbonne - Paris III, dans sa présentation de la conférence, du 20 juin 2018 au 22 juin 2018, titrée "Gayatri

(Texte et paratexte dans ...) Ibrahim Abouelmaati Ibrahim Elmorsy 
Spivak dans le siècle. Politiques de lecture et écriture pour refonder une imagination critique Autour des travaux de Gayatri Spivak." souligne le rapport entre les deux : "sans oublier le rapport singulier de Spivak à l'œuvre d'Assia Djebar"(24)

L'emplacement canonique de la dédicace est normal d'après le point de vue de Genette; elle est placée après la page de titre. Ainsi se présente la dédicace du roman:

"à Gayatri,

Avec mon affection." NMP., p.7;

Assia Djebar a voulu rendre hommage à cette écrivaine pour la totalité de son œuvre et pour ses idées très proches d'elle.

On a aussi une donnée paratextuelle que l'auteure a placée au début de son roman, mais elle est clairement séparée de lui: c'est l'épigraphe qui vient normalement après la dédicace :

"De loin je suis venue et je dois aller loin...

\section{KATHLEEN RAINE,}

"Le Voyage", The Pythoness, 1949" NMP., p.9

Cette épigraphe nous étonne comme lecteurs, elle est tirée d'un poème dont le titre anglais est traduit par Assia Djebar, c'est 'Le Voyage" qui est à son tour tiré d'un recueil nommé "The pythoness", de Kathleen Jessie Raine qui est une poétesse britannique. Djebar a écrit non seulement le nom et le prénom de cette écrivaine en lettres majuscules mais aussi tous les intertitres du roman, à l'exception des intertitres concernant les trois parties, et tout cela pour les mettre en relief et aussi pour souligner l'intérêt et la valeur de ces événements narrés, de ces personnes rencontrées et de ces lieux fréquentés dans sa vie soit privée, soit publique. Il est remarquable que l'épigraphe a été traduite en français par l'auteure. En se plongeant dans le roman, on découvre

(Texte et paratexte dans ...) Ibrahim Abouelmaati Ibrahim Elmorsy 
qu'Assia Djebar nous renseigne sur son intérêt immense pour la lecture et les livres : "Lectrice de tant de romans, de poèmes, tant de chroniques(...) grâce à cette passion qu'entretenait en moi la faim dévorante et nocturne des livres, que s'approfondissait peu à peu le cours de ma maturation." NMP, p.274 ; et ça va être affirmé à travers la dédicace ou l'épigraphe. D'après Genette, l'épigraphe : " est à elle seule un signal (qui se veut indice) de culture, un mot de passe de l'intellectualité. "(25)

Dans cette épigraphe très simple, Djebar exprime son passé et aussi son avenir, elle s'attache étroitement à ses ancêtres, à tout lieu lointain dans l'Histoire de sa famille; Assia Djebar souligne les deux mondes dans lesquels elle a vécu: celui de l'enfance (algérien) et celui de l'enseignement (européen): " à force de vivre parmi les femmes voilées, masquées, calfeutrées sous la laine en quelque sorte "non vue"(...) à l'autre monde, celui du lycée, de la pension, de nos professeurs et même des gens de la rue, visible à ce monde "européen"'. NMP., p.285

\section{Les intertitres :}

À l'intérieur du roman choisi, on trouve des titres intérieurs ou internes, titres des parties et aussi ceux des sections, que Gérard Genette appelle intertitres. On a trois parties titrées et dont chacune se compose de plusieurs sections numérotées et titrées aussi. Gérard Genette souligne la différence majeure entre le titre générale de l'œuvre qui devient "un élément indispensable sinon à l'existence matérielle du texte, du moins à l'existence sociale du livre"; et les intertitres que leur présence à l'intérieur du livre n'est "nullement une condition absolue."(26)

D'après Genette, il y a des degrés ou du moins des modalités de présence des intertitres : des régimes thématiques (le titre du chapitre seulement), rhématiques (le numéro du chapitre seulement) ou mixte (le titre et le numéro) ${ }^{(27)}$; dans le roman à

(Texte et paratexte dans ...) Ibrahim Abouelmaati Ibrahim Elmorsy 
étudier, on trouve le régime mixte où l'auteure présente les deux modalités. "Nulle part dans la maison de mon père" est un roman exceptionnel par ses données paratextuelles. On est étonné par la division intérieure du roman qui lui donne une qualité autre que les autres romans d'Assia Djebar, ça revient peut-être à la grande quantité des informations données par l'auteure concernant sa vie privée, ou peut-être à la place de cet ouvrage dans l'itinéraire romanesque djebarien.

La première partie, intitulée "Éclats d'enfance", contient neuf sections numérotées; la deuxième, qui porte comme titre " Déchirer l'invisible", se compose de douze sections, aussi numérotées ; et la troisième, titrée "Celle qui court jusqu'à la mer", renferme onze sections, également numérotées.

Ces intertitres contribuent fortement à éclairer plus ou moins les phases variées de la vie de l'auteure, chaque titre est tout-à-fait lié à l'un des événements de sa biographie. La relation étroite entre le texte et le paratexte apparaît clairement à travers "l'appareil intertitulaire $^{\text {n(*) }}$ du roman ; chaque intertitre s'attache à ce que l'auteure raconte, sans oublier la part fictive de la romancière. Expliquons maintenant les intertitres du roman :

\section{La première partie du roman "Éclats d'enfance":}

Dans cette partie du roman, la narratrice raconte ses souvenirs d'enfance, normalement attachés à ses parents, à ses grandsparents, à l'école coranique ; elle subit d'ailleurs toutes les restrictions d'une société traditionnelle.

\section{Le premier intertitre "1. LA JEUNE MÈRE " :}

(Texte et paratexte dans ...) Ibrahim Abouelmaati Ibrahim Elmorsy 
Dans cette section, la narratrice parle de sa mère, petite et encore soumise aux traditions des ancêtres, en exprimant son affection à l'égard de cette jeune femme : "Toute jeune, enveloppée de pied en cap dans un voile de satin blanc (...) ma mère se couvre lentement du haïk (...) La marcheuse est ensevelie sous la soie immaculée, elle dont on ne pourra apercevoir que les chevilles et, du visage, les yeux noirs..."NMP., pp.14-15; à travers cette description, Djebar indique la tenue vestimentaire de la femme algérienne, durant la première moitié du XXe siècle, qui était obligée de mettre le voile en sortant de chez elle.

\section{Le deuxième intertitre " 2. LES LARMES " :}

Dans cette section, la narratrice indique sa première lecture d'un roman titré "Sans Famille", écrit par Hector Malot ${ }^{(*)}$. C'est un conte très émouvant concernant la famille qui traite la souffrance d'un enfant loin de sa maison paternelle ; la narratrice est si saisie par les événements du roman et la condition de cet enfant abandonné en l'absence totale de famille. Alors, la narratrice commence à pleurer fortement si bien que ses parents l'observent dans un état déplorable: "La fillette pleure devant ce malheur" NMP., p.20; le souvenir de ce conte dure longtemps dans la mémoire de la narratrice qui fait, après le passage de plusieurs années, une petite comparaison entre sa situation et celle de cet enfant "le héros du roman" : " elle se demandera si ces larmes évoquées ne tenaient pas leur douceur du lit de ses parents où elle s'était jetée, alors que le garçonnet du livre, lui, ne connaissait nul repos, nul havre dans ses malheurs tout au long des pages tournées." NMP, p.21

Mais ce n'était pas la première fois dans laquelle la narratrice a pleuré, elle raconte l'événement douloureux de la mort de sa grand-mère paternelle, alors qu'elle a presque trois ans : "mes larmes en flux bouillonnant" NMP., p.25; Dans une phrase très

(Texte et paratexte dans ...) Ibrahim Abouelmaati Ibrahim Elmorsy 
courte, la narratrice exprime sa douleur et ses chagrins : "Mamma m'a abandonnée" NMP., p.24, cette grand-mère représente alors la tendresse et l'amour pour la narratrice, en l'absence de la tendresse paternelle : "Personne ne m'aimera, que ma mère." NMP., p.24 "les mains de ma grand-mère, la nuit, me caressant contre le froid, ces mains, où les trouver..."NMP., p.25

Il est clair que les larmes de la narratrice ne cessent et se poursuivent plus tard vers la fin de la période de l'enfance, elle dit:" mes larmes couleraient encore, mais douces à cause de cette distance en années, en décennies multipliées." NMP., p.30; la sensation d'être emprisonnée dans la maison de son père, surtout durant les vacances d'été pousse encore une fois la narratrice à pleurer.

\section{Le troisième intertitre "3. LE TOUT PREMIER LIVRE" :}

À travers cette section, la narratrice raconte l'histoire de son premier prix obtenu comme prix d'excellence de l'école ; mais elle remarque que son père n'était pas content : "ce qui me frappe, c'est l'étrange demi-sourire sur la face paternelle." NMP., p.33 et elle a su la raison; mais plus tard lors d'une conversation avec son père :" Je n'étais pas pétainiste." NMP., p.35; parce qu'au-dessus de la première couverture du livre se trouve la tête du maréchal Pétain qui dirige alors la France. De toute façon, "On ne peut mettre en doute l'engagement d'Assia Djebar, comme celui de sa famille (...) le père était déjà engagé dans la lutte contre l'injustice coloniale, avant de l'être, encore plus, contre la misère des ouvriers algériens." ${ }^{(28)}$ a déclaré Zineb Ali-Benali ; dans cette section, elle jette la lumière sur sa vie à l'école "c'est moi la première" NMP., p.33, et aussi l'aspect politique de sa famille.

Dans cette section, on a un sous-titre :'Intermède" où la narratrice cherche une définition du mot "Colonie" en essayant d'indiquer la présence coloniale dans son pays d'origine. Elle a

(Texte et paratexte dans ...) Ibrahim Abouelmaati Ibrahim Elmorsy 
voulu exprimer non pas seulement son déchirement intérieur, mais aussi celui d'un peuple tout-entier, en faisant allusion à l'une des significations voulues par le titre du roman :" La colonie est un monde sans héritiers, sans héritage. Les enfants des deux bords ne vivront pas dans la maison de leurs pères. "NMP., p.37; dans cette partie, la narratrice souligne une enfance divisée et tiraillée entre deux mondes qui coexistent ensemble, en dévoilant l'héritage laissé par les ancêtres :" La rancune en partage, au mieux l'oubli, le plus souvent le désir de partir, de fuir, de renier, de chercher n'importe quel horizon..." NMP., p.37-38

\section{Le quatrième intertitre "4. LE PÈRE ET LES AUTRES":}

Dans cette section, la narratrice donne un portrait de son père, à la fois physique et moral, en indiquant une part de ses relations en famille et hors de famille. Elle décrit la sévérité de son père même avec les autres : " un soir, en famille- son verbe, en français, devint rude- il nous rapporta (...) comment la veille un père d'élève-un Français, un Espagnol ou un maltais-s'était permis de le tutoyer à la porte de l'école parce que cet "instituteur arabe (pensait l'Européen) est décidément plein de morgue..." NMP., p.47

La narratrice se rappelle son départ pour Paris et sa rencontre avec l'un des amis de son père, qui lui parle de la grand-mère paternelle. Elle poursuit la description de son père : "Mon père était un jeune homme très grand, aux larges épaules. Il avait les yeux bleu-vert de son père ou peut-être de cette grand-mère maternelle." NMP., p.47

\section{Le cinquième intertitre "5. LA BICYCLETTE":}

Dans cette section, la narratrice raconte une scène très importante, voire très choquante quand elle était encore petite ; c'est celle de la bicyclette. elle veut monter à vélo en compagnie d'un garçon

(Texte et paratexte dans ...) Ibrahim Abouelmaati Ibrahim Elmorsy 
français et son père l'a vue. Elle indique à quel point son père était un homme sévère, il refuse toujours la promiscuité ; mais sa réaction inattendue à la maison face à sa mère laisse des traces inoubliables dans la mémoire de la narratrice, alors déçue et étonnée :" je ne veux pas, non, je ne veux pas - répète-t-il haut à ma mère, accourue et silencieuse -, je ne veux pas que ma fille montre ses jambes en montant à bicyclette!" NMP., p.55; la narratrice commence à se poser des questions : "Mon père est-il le même? Peut-être devient-il soudain un autre? (...) Qu'est-ce que cela veut dire: sa phrase, son ton, sa colère, le fait que pour la première fois, il se rue dans "leur" chambre, cet antre ?" NMP., p.55

\section{Le sixième intertitre "6. LE JOUR DU HAMMAM":}

Assia Djebar jette quelques lumières sur un lieu remarquable dans l'Histoire des pays du Maghreb, c'est le hammam dont la narratrice indique l'importance pour toutes les femmes : "L'aprèsmidi au bain, pour elles, constitue l'événement de leur semaine. Elles ont dû arriver tôt, dès l'ouverture de la séance pour femmes, vers les onze heures du matin." NMP., p.68

La narratrice décrit le lieu et aussi sa responsable ; autrement dit la gérante : "dans la salle froide de l'entrée du hammam, au fond d'un coin sombre avec estrade, est réservé un lieu où sont installés des divans confortables et où s'amoncellent des matelas couverts de tapis aux vives couleurs (...) Lla Aïcha, la gérante des lieux (...) a salué ma mère (...) Lla Aïcha, si démonstrative..." NMP., pp.67-68

Ce lieu représente également une source très importante des informations ; en parlant de la gérante, la narratrice souligne qu'elle "converse à voix menue avec ma mère ; elle lui donne des nouvelles des deux ou trois familles "connues" du village." NMP., p.71; la narratrice décrit sa mère pendant son aller-retour pour le

(Texte et paratexte dans ...) Ibrahim Abouelmaati Ibrahim Elmorsy 
hammam, au centre du village comme à la ville, elle est: "totalement masquée." NMP., p.79; malgré cela, elle n'est pas à l'écart des regards des autres, surtout "les Européens installés aux terrasses de leurs brasseries." NMP., p.79

\section{Le septième intertitre "7. LE PETIT FRÈRE" :}

La narratrice souligne dans cette section les souvenirs de la mort de son petit frère et la tristesse de sa mère :" La mort du premier frère, un bébé de six mois." NMP., p.83; Assia Djebar donne ici à son lecteur une information autobiographique concernant le nom réel de père à travers la parole de la mère de la narratrice adressée à son père:" O Tahar (...) nous étions quatre (...) nous voici revenus à trois!" NMP., p.85

La narratrice indique également que pendant sa présence à Paris avec sa cadette, elle se rappelle le changement qui s'est passé dans la mentalité de son père et aussi dans les habitudes de sa mère. Au fil du temps, le père a accepté que sa cadette, devenue plus tard une femme médecin, monte à vélo et la mère vit comme les européennes : "Retournons donc au village colonial lorsque la benjamine du couple, pour revivre son enfance plus "libre", en tout cas moins frappée d'interdits que la mienne-huit ans nous séparent, années d'évolution rapide(...) ma sœur, je crois, apprendra sans encombre à enfourcher une bicyclette, verra sa mère(...) se mettre même à sortir en Européenne, c'est-à-dire sans le voile islamique." NMP., p.99

\section{Le huitième intertitre "8. DANS LA RUE? AVEC LE PÈRE? OU JEUX DE MIROIRS":}

Dans cette section, la narratrice souligne comment son père s'était occupé de l'apprendre la langue du colonisateur dans une école française et le Coran à travers le maître coranique "le cheikh" dans une médersa; alors que les autres filles de son âge sont privées de cette qualité, c'est la part positive du père : "Je suis

(Texte et paratexte dans ...) Ibrahim Abouelmaati Ibrahim Elmorsy 
fière de la fierté de ma mère, tandis que je récite la sourate aux invitées qui m'envient (leur père ne leur a permis ni l'école française, ni même l'école coranique)" NMP., p.99

D'ailleurs, elle raconte son allée à l'école coranique en compagnie de son père qui prend sa main le matin seulement : "je vais deux ou trois fois par semaine" NMP., p.97; mais en retournant à la maison, le père, regardé alors par les autres, s'attache à ses croyances traditionnelles : "Un homme arabe, père de famille, doit marcher seul, son regard posé sur ses enfants." NMP., p.100 Le lieu où elle apprend le coran était l'arrière-boutique d'un épicier. Elle explique également l'importance de l'apprentissage de la langue française, et surtout pour son père qui lui dit: " c'est pourtant la langue, celle des autres, qui reste son armure..." NMP., p.97

La narratrice indique aussi la situation de sa mère après la mort de son père, elle la décrit comme " une chatte sans menottes" NMP., p.104; et la mère dit à sa fille en exprimant son amertume pour la perte de son mari, et en avouant ses sensations envers lui: "Reçois-moi, moi, l'amante de ton père, reçois-moi comme un bébé qui vient de naître." NMP., p.105.

\section{Le neuvième intertitre "9. LA CHAMBRE PATERNELLE":}

La narratrice parle de la chambre paternelle où elle s'endort jusqu'à la naissance de son petit frère. Âgée alors dix-huit mois, la narratrice va dormir avec sa grand-mère paternelle. Elle nous dit que : " tout bébé, si petit soit-il, me dis-je dans une réminiscence, ne doit pas dormir si près du lit parental." NMP., p.112

Ainsi, la première partie du roman " éclats d'enfance" est tout-àfait attachée à cette période de l'enfance de la narratrice, dès ses premières années en passant par l'école coranique jusqu'à son allée à l'école ; elle est encore une fillette.

(Texte et paratexte dans ...) Ibrahim Abouelmaati Ibrahim Elmorsy 


\section{La deuxième partie "Déchirer l'invisible" :}

Dans cette partie, composée de douze sections, la narratrice aborde une autre période de sa vie : c'est l'adolescence.

\section{Le premier intertitre "1. MADAME BLASI":}

Dans cette section, la narratrice dépasse la période de l'enfance pour expérimenter celle de l'adolescence. Madame Blasi est son institutrice française à l'internat, elle la décrit comme :"première Française à m'avoir fait un tel don, par élan, par recueillement, comme à toute la classe silencieuse, une classe de sixième de lettres classiques." NMP., p.116

La narratrice a découvert la beauté de la langue française à travers Baudelaire et son poème L'invitation au voyage, qui est devenu pour elle comme "l'invitation à la beauté des mots français..." NMP., p.117

D'une part, la narratrice jette la lumière sur son intérêt à sa langue maternelle "l'Arabe" qu'elle a apprise seulement à travers l'école coranique ; et d'autre part, elle apprend la langue française d'une manière totalement différente, à travers la littérature française, et surtout la poésie et les poètes. Djebar a voulu peut-être justifier son choix d'écrire en français, la langue du colonisateur. Elle appartient à une génération qui maîtrise bien le Français et non l'Arabe. La narratrice dit: " je voudrais apprendre littéralement la langue de ma mère, celle de mes aïeux-par ses poètes et ses textes anciens, et non comme au village où j'allais à l'école coranique et où le coran s'apprend par cœur, donc sans vraiment comprendre!" NMP., p.118

\section{Le de uxième intertitre "2. PREMIERS VOYAGES ? SEULE...":}

Cette section raconte le voyage entrepris par la narratrice chaque semaine, précisément chaque samedi, de l'internat au village natal; "la pensionnaire libre mais prudente" NMP., p.124 arrive à

(Texte et paratexte dans ...) Ibrahim Abouelmaati Ibrahim Elmorsy 
la station des cars après moins de vingt minutes de marche. Là, elle était saisie par le paysage et les gens. Elle a pris le car qui se dirige vers son village natal. Pendant le retour, la narratrice voit beaucoup de gens, un amalgame de nationalités qui coexiste ensemble. Dans le même car, on trouve:" Une Espagnole(...) deux dames voilées(...) paysans berbères(...) Quelques employés d'allure européenne." NMP., pp.125-126; la narratrice commence alors son premier voyage seule sans être accompagnée ni de père ni de mère, pour cela, elle était si attentive de tout ce qui l'entoure:" Enfin le car démarre, et moi, (...) je ne veux rien perdre du paysage." NMP., pp.126-127

À travers ce voyage, la narratrice souligne à quel point son père, malgré son absence, était au courant de tout ce qui se passe autour de sa fille, il surveille bien ses pas; pendant que la fille attend le départ du car, elle nous raconte l'épisode:" l'employé qui connaît mon père est venu vérifier où je me trouvais" NMP., p.125; et le père à son tour va attendre l'arrivée de sa fille: "à peine la porte du car s'est-elle ouverte (...) mon père, d'un simple pas en avant, se dresse devant moi, me tend la main et me sourit." NMP., p.130 Cette rencontre entre les deux témoigne de l'austérité du père qui respecte les traditions anciennes en cachant ses émotions envers sa fille pour sauvegarder son image comme chef de la famille : "chaque fois, d'instinct, je sais que je dois me retenir de ne pas lui sauter au cou; nous sommes dehors, devant tous; or, chez les "nôtres", on n'embrasse pas les deux joues paternelles (mon père ne se serait d'ailleurs pas incliné). NMP., p.130

\section{Le troisième intertitre "3. LE PIANO":}

La narratrice raconte ici son expérience avec l'apprentissage à jouer du piano à l'internat, et aussi comment sa mère s'attache à la musique traditionnelle andalouse, et ses efforts déployés pour que sa fille réussisse à apprendre le piano :" Est-ce cette année-là

(Texte et paratexte dans ...) Ibrahim Abouelmaati Ibrahim Elmorsy 
qu'elle propose à mon père de m'acheter un piano ? Il est assez vite d'accord pour payer le professeur de solfège." NMP., p.134; la narratrice, après avoir assisté à un concert à l'internat pour la première fois, a été très ravie d'écouter cette musique occidentale : " un concert programmé par les Jeunesses musicales : le célèbre Samson François, venu de Paris, doit jouer plusieurs œuvres de Chopin." NMP., p.135; mais elle n'a pas réussi, c'est peut-être à cause de la professeure qu'elle décrit comme "dame sèche et revêche" NMP., p.141, " trois ans, au moins, avait duré cette tentative qui se soldait par un échec." NMP., p.143

Il est remarquable que son échec à apprendre le piano l'a poussée à s'intéresser seulement aux livres, la narratrice dit que : " $L a$ lecture sera mon ivresse! La seule..."NMP., p.142

\section{Le quatrième intertitre "4. LA PREMIÈRE AMIE":}

La narratrice parle ici des pensionnaires dans l'internat où elle rencontre des filles européennes et des autres indigènes (c'est-àdire des filles algériennes soit de Kabylie, soit d'autres lieux de son pays). En parlant des filles musulmanes dans l'internat, la narratrice dit que : "leurs commentaires me rappelaient les bavardages des villageoises au bain maure, que ma mère, autrefois, dédaignait." NMP., p.146. Durant la première année du séjour au pensionnat, la narratrice n'aime s'approcher ni des fillettes françaises, ni des fillettes arabes ; mais la seconde année assiste à la connaissance d'une fillette étrangère "Mag" d'origine italienne, et toutes les deux partagent la passion pour la lecture. Parmi leurs lectures, on trouve "la Correspondance de Jacques Rivière et d'Alain-Fournier" NMP., p.147, la discussion entre les deux pensionnaires sur les livres lus était forte, et la narratrice donne la synthèse de sa lecture en critiquant le colonisateur : "ironie du sort, car, dans cette petite ville coloniale, la modernité était pratiquement inconnue." NMP., p.147

(Texte et paratexte dans ...) Ibrahim Abouelmaati Ibrahim Elmorsy 
La présence de Mag dans la vie de la narratrice joue un rôle primordial, c'est celle qui l'a poussée dans l'univers littéraire, en l'aidant à se débarrasser de l'autorité paternelle, elle représente alors sa vraie amie, la seule qui l'a fait sortir de sa "solitude et du confinement provincial." NMP., p.149

\section{Le cinquième intertitre "5. FARIDA ? LA LOINTAINE":}

La narratrice poursuit la parole concernant sa vie comme pensionnaire musulmane, différente des autres filles européennes dans le même internat. Elle découvre Farida, demi-pensionnaire, fille unique d'un officier musulman de l'armée française, qui a subi des contraintes journalières très dures : " m'étonnais-je devant une telle rigueur (...) Ainsi, mon père, qui semblait jusquelà si sévère, s'avérait plus libéral que celui de Farida." NMP., p.163

\section{Le sixième intertitre "6. AU RÉFECTOIRE":}

Dans cette section, la narratrice indique comment au réfectoire les pensionnaires étaient divisées en deux groupes : " à cause de nos tabous alimentaires." NMP., P. 171 ; en soulignant la situation avantageuse des Françaises:" On nous donne chaque fois deux oufs au plat avec de la purée. Or, les Françaises ont droit, elles, à de la viande !" NMP., p.173

La narratrice n'oublie jamais la croyance religieuse à laquelle s'attache les pensionnaires algériennes, elle parle du mois de Ramadan, le plus favori chez tous les musulmans, c'est le mois du jeûne. Elle dévoile comment la vie à l'internat était difficile durant ce mois-ci, et cela revient à la différence des rites religieuses: "nous jeûnions donc, nous, les musulmanes, dans la journée, tout en suivant les cours." NMP, p. 183

\section{Le septième intertitre "7. LE MONDE DE LA GRAND- MÈRE MATERNELLE":}

(Texte et paratexte dans ...) Ibrahim Abouelmaati Ibrahim Elmorsy 
La narratrice traite dans cette section sa relation avec sa grandmère maternelle qu'elle décrit ici comme une dame sévère, qui préfère la fille de son fils unique sur elle. Cette dame garde aussi la langue berbère, celle des ancêtres, pendant sa conversation avec ses métayers. La narratrice indique son ignorance de cette langue:" La mère de ma mère était cette orgueilleuse veuve (...) dans une langue que je ne comprenais pas (...) C'était en langue berbère que la maîtresse de maison communiquait avec ce paysan." NMP., p.185; ainsi, Assia Djebar insiste toujours sur la présence de la langue berbère dans sa vie, ou plutôt dans la vie de tout Algérien.

La narratrice affirme l'enfermement dans lequel vit les filles qui sont : "Enfermées comme internes durant l'année scolaire, puis, l'été, séquestrées comme nos mères." NMP., p.189; la narratrice se souvient des vacances d'été qu'elle passées chez sa grand-mère maternelle, en soulignant aussi la différence entre la vie citadine chez elle et la vie rurale chez son père, : "un été où se succédaient les noces (...) Mère et moi, nous vivons dans village (...) nous n'avons guère de distractions!" NMP., p.191

\section{Le huitième intertitre " 8. JACQUELINE...AU DORTOIR...":}

La narratrice jette la lumière sur sa vie au dortoir avec une fille française, Jacqueline qui est sa voisine de lit; elle la voit " pleine de liberté" NMP., p.195.

La narratrice essaie toujours durant sa présence à l'internat de souligner la présence de deux mondes tout-à-fait différents, celui de son père et celui du colonisateur. Deux mondes coexistent ensemble et dont chacun a ses pratiques sociales et ses rites religieux différents. Pour elle, Jacqueline appartient à " un monde

(Texte et paratexte dans ...) Ibrahim Abouelmaati Ibrahim Elmorsy 
aux mours bien étranges!" NMP., p. 195. La narratrice souligne également qu'elle était toujours entourée du regard de son père, au moment où les filles françaises jouissent de sa vie pleine de liberté sous le regard de leur famille, la protagoniste parle de soimême en disant : "'fillette sage" qui sentait posé sur elle, malgré elle, ou peut-être la raidissant, le regard du père au discours souvent parsemé de souvenirs sur lui-même." NMP., p.195; tandis que sa camarade Jacqueline la raconte une scène avec un flirt, la narratrice se rappelle ces mots: "les Françaises ignorent toute pudeur." NMP., p.196 Devant toute cette liberté immorale de Jacqueline et sa transgression, aux yeux de la narrataire, elle se remémore sa cousine qui va se marier en la jugeant à sort favorable : "elle a de la chance - inestimable dans notre sociétéde connaître son mari, puisqu'il est son cousin." NMP., pp.199200 , quelle divergence!

\section{Le neuvième intertitre "9. CORPS MOBILE":}

Le corps de la narratrice, thème repris à plusieurs reprises dans le roman, est pour le père une chose sacrée qui doit être cachée des regards des autres; et cela est affirmé par l'épisode de la bicyclette, déjà cité durant la période de l'enfance de la narratrice. À l'internat, le corps de la protagoniste a trouvé un lieu spacieux, même entouré d'une clôture très haute, qui le pousse à être mobile. C'est le terrain de basket-ball qui représente pour la narratrice un lieu immense et aussi un espace de liberté : " sur ce stade, ma liberté m'inonde, corps et âme, telle une invisible et inépuisable cascade." NMP., p.203.

En indiquant la sévérité de la société dans laquelle elle vit, surtout à l'égard des femmes qui sont menottées par des règles fermes, la narratrice, alors jeune fille et non une grande fillette, raconte sa tentative de choisir une robe dont le dos et les épaules sont nus pour se préparer aux cérémonies familiales : "Trois ou

(Texte et paratexte dans ...) Ibrahim Abouelmaati Ibrahim Elmorsy 
quatre mariages sont annoncés parmi la parentèle et les alliées; deux ou trois circoncisions." NMP., p.212, la mère, après son accord hésitant, avait peur de son mari; ce petit dialogue entre les deux exprime ce que ressent la mère et aussi la relation intime entre la mère et la fille :

"- Je te promets, Mma : jamais les épaules nues devant mon père, avec cette robe! Il ne verra rien!

- Ni dehors, n'est-ce pas?

- Ni dehors (je l'étreins), ni dans la rue!" NMP, p.212

Vient enfin la description de sa danse qui représente une expression de la liberté du corps longtemps prisonnier dans la tenue vestimentaire traditionnelle, la narratrice raconte cette expérience inoubliable :" je danse d'abord lentement comme une paonne, puis légèrement ensuite, à gestes déliés, comme une almée, pour finir, nerveuse, tressaillant des épaules, rendant lianes mes bras nus sur le point de se coucher au sol..." NMP., p.214

\section{Le dixième intertitre " 10. L'OPÉRETTE":}

La narratrice raconte dans cette section comment l'internat organise à la fin d'année scolaire des fêtes au mois de juin, c'est un événement extraordinaire pour la narratrice où se rassemblent le lycée de garçons et le collège de jeunes filles pour la coproduction d'une opérette. Le programme de cette fête contient "l'existence d'une opérette, "Les Cloches de Corneville" "*), qui avait remporté (...) un succès mondial depuis sa création...en 1877!(...) je fus choisie pour faire partie du chour des paysannes." NMP., pp.226-227

\section{Le onzième intertitre " 11. UN AIR DE NEY":}

Dans cette section, la narratrice parle du livre dont elle a lu la traduction à bord d'un avion qui la transporte d'Alger à Paris; alors

(Texte et paratexte dans ...) Ibrahim Abouelmaati Ibrahim Elmorsy 
elle est de quarantaine. Ce livre titré "Mathmawi ou les odes mystiques" est l'ouvrage majeur de Jalal al-Din Rûmi, poète mystique persan qui est né en 1207. La narratrice traite l'histoire de ce livre qui exprime un récit allégorique de Ney, celle-là lui donne le titre de la deuxième partie : "Dans cette æuvre poétique, Jalal al-Din Rûmi nous dit que "la première chose créée par Dieu a été la plume du roseau", puis il illustre cette assertion par une histoire(...) elle justifiera le titre qui s'est imposé à moi pour cette traversée de mon adolescence: "Déchirer l'invisible"".. NMP., p.261; la narratrice décrit la période de l'adolescence comme une belle mélodie :" oui, telle cette adolescence aussi belle qu'un air de ney." NMP., p.261

A la fin de cette section, la narratrice se pose une question exprimant son destin inconnu en traversant cette période de vie qu'elle cherche à déterminer sa forme; la narratrice répète pour la deuxième fois dans la même section le titre de la deuxième partie" Déchirer l'invisible", mais en renversant sa syntaxe, comme si elle veut dire que l'invisible du passé est maintenant disponible et prêt à être visible :" à quelle transmission ou à quelle métamorphose ai-je été destinée dans cet invisible à déchirer, tel que j'ai désiré l'esquisser?" NMP., p.266

\section{Le douzième intertitre "12. L'ÉTÉ DES AÏEULES...":}

Dans cette dernière section de cette partie, la protagoniste ressuscite le passé avec joie : "je me contente de suivre le rythme des réminiscences." NMP., p.267; mais elle est complètement très influencée par ses vacances passées chez sa grand-mère maternelle : "les traces d'enfance, les remous de l'adolescence avaient soudain été recouverts par l'image démultipliée ou disloquée de la grand-mère maternelle." NMP., p.269 ; la narratrice se reconnaît :" oui, c'est de cette aïeule que je suis la

(Texte et paratexte dans ...) Ibrahim Abouelmaati Ibrahim Elmorsy 
descendante (...) je suis son héritière à ma manière brouillonne." NMP., pp.269-270

\section{La troisième partie " Celle qui court jusqu'à la mer":}

Dans la troisième et la dernière partie du roman, composée de onze sections numérotés, la narratrice exprime sa transition de l'adolescence à l'âge adulte.

\section{Le premier intertitre " 1. ENCORE AU VILLAGE":}

La narratrice parle ici du déménagement de sa famille pour s'installer à la capitale d'Algérie, en la nommant: "Alger-lasultane" NMP., p.273; puis son allée pour vivre dans des capitales nombreuses : " à Paris, à Tunis et à Casablanca" NMP., p.273. La narratrice ne peut pas s'éloigner de ses livres qui représentent sa seule évasion : "Je continuais à lire de nuit (...) la lampe de poche sous le drap, au dortoir." NMP., p. 274

Malgré sa présence à Alger, la narratrice est encore attachée à son village d'enfance par ses mémoires inépuisables, elle revit là avec ses souvenirs; elle se rappelle tout :" Revenant sur ces derniers jours de printemps au village, je me remémore ces spectateurs tapis dans l'ombre du kiosque, j'imagine à leur suite leurs femmes, elles que je rencontrais enfant, lorsque j'allais au bain maure avec ma mère." NMP., p.277; elle termine cette section en disant :" Mais je ne suis plus la fillette au village." NMP., p.278

\section{Le deuxième intertitre "2. LETTRE DÉCHIRÉE":}

La narratrice raconte un épisode très important dans sa vie, encore au village et encore adolescente, celui-ci est relatif à une lettre envoyée à elle par un inconnu et la réaction de son père qui : " $a$ mis la lettre en mille morceaux(...) je suis restée abasourdie devant la violence de la colère paternelle." NMP., p.279 ; mais le silence de la narratrice ne dure pas longtemps, elle a le courage de reconstruire les débris de la lettre jetée à la corbeille par le père;

(Texte et paratexte dans ...) Ibrahim Abouelmaati Ibrahim Elmorsy 
alors elle éprouve un sentiment de culpabilité, elle s'avoue :" Mon premier péché est donc de curiosité: lire la missive qui a déclenché la rage de mon père (...) Mon second péché fut de désobéissance prémédité (...) Mon troisième péché fut ainsi de paraître user des stratagèmes, en intrigante avertie." NMP., pp.280-281

Ainsi, la narratrice accepte de correspondre avec cet inconnu; au début, elle le nomme "l'étudiant d'Alger" NMP., p.280; ensuite elle a connu son prénom "il se prénommait Tarik" NMP., p.286

\section{Le troisième intertitre " 3. PREMIER RENDEZ-VOUS":}

Dans cette section, la narratrice se remémore sa première rencontre avec Tarik à Alger, loin de son village :" c'était un jour de l'automne ou de l'hiver 1952." NMP., p.291; tout au long de cette section, elle parle de Tarik et aussi le décrit en parlant de ses origines turques. Elle décrit aussi cette expérience en disant: "première audace de mes quinze ans, me voici marchant ainsi "accompagnée" à mon tour, moi, la musulmane, par un jeune homme certes inconnu, mais, à tout le moins, de ma communauté, et cela, malgré la fausse aisance que j'affichais, devenait vraiment d'une dangereuse hardiesse!" NMP., p.303

\section{Le quatrième intertitre " 4. LETTRES DITES D'AMOUR":}

Ici la narratrice se rappelle son courage de poursuivre une correspondance clandestine avec Tarik qui a envoyé ses lettres au collège en employant un prénom d'une jeune fille "Béatrice", pseudonyme inventé pour cacher la véritable identité de la destinatrice, au dos de l'enveloppe. Dans ses lettres, Tarik traite "la célèbre poétesse el Khansa" NMP., p.317, en donnant les deux versions : le texte original et la traduction française. Il parle aussi de "Nabigha al-Dhubyani, l'un des dix - ou des sept - auteurs des Mo'allaquats les plus prestigieuses." NMP., p.319; la narratrice

(Texte et paratexte dans ...) Ibrahim Abouelmaati Ibrahim Elmorsy 
s'avoue aussi :" je reçus de lui un vrai poème d'amour, d'une si bouleversante beauté que je ressentis, en moi, une émotion prolongée." NMP., p.325

La narratrice exprime finalement son audace de recevoir les salutations de Tarik devant tous, durant sa présence à la fête organisée par le collège pour la distribution des prix, bien sûr en l'absence de son père. $C f$., $N M P$., p.327

\section{Le cinquième intertitre " 5. LA FAMILLE À ALGER":}

La narratrice se rappelle encore une fois dans cette section le départ de sa famille pour Alger, c'est le père qui décide cette affaire :"mon père nous annonça qu'il était nommé, pour la rentrée suivante, à la capitale." NMP., p.329

La narratrice parle du changement inattendu qui s'est passé en sa famille au cours de sa présence à Alger, la jeune fille elle-même a obtenu l'accord de son père de poursuivre plus tard ses études supérieures à la faculté, elle décrit alors l'évolution :" minirévolution, donc, au sein de ma famille appartenant à la bourgeoisie traditionnelle de Césarée et restée auparavant à l'écart de la société européenne du village de colonisation, durant plus de quinze ans!" NMP., p.329

Même sa mère n'était pas exclue de cette mini-révolution familiale, de ce bouleversement radical, la narratrice observe heureusement cette métamorphose inattendue :" ma mère, qui allait fêter ses trente-six ans, se métamorphosa en quelques mois en occidentale d'une élégante discrète." NMP., p.330

\section{Le sixième intertitre "6. DANS LA RUE":}

La narratrice souligne ici comment cette vie nouvelle à Alger l'a rendue coupable aux yeux de ses indigènes :" L'impudique! Sans voile, et pas même les cheveux dissimulés!" NMP., p.337

(Texte et paratexte dans ...) Ibrahim Abouelmaati Ibrahim Elmorsy 
La jeune fille était passionnée d'Alger, c'est un autre monde, totalement différent de celui du village ; même au niveau de la langue parlée: "Dans la rue, alors que je peux laisser mon corps vagabonder, libre, il me faut me taire ou bien parler français, anglais, et même chinois si je pouvais. " NMP., p.340

\section{Le septième intertitre "7. PROMENADES AU PORT":}

Malgré son bonheur d'être à Alger, d'être promeneuse de cette ville charmante, et malgré sa croyance qu'elle a l'air européenne en maitrisant le Français, elle a découvert la vérité avec le premier contact quand elle a voulu déchiffrer l'invisible dans cet univers ; une fois, en sortant du lycée, la narratrice décrit son état d'âme et raconte aussi sa déception :" Je m'arrêtais devant une porte basse pour en soulever le heurtoir (...) parler à l'hôtesse en arabe, lui demander de me laisser admirer le patio ancien, les mosaïques aux couleurs pâlies; avec nonchalance, elle me faisait entrer (...) je retournais aux rues dites "européennes", comme si j'étais devenue une véritable étrangère, partout dans cette capitale." NMP., pp.348-349

La jeune fille se rappelle encore sa promenade avec Tarik, celui qu'elle désigne comme son fiancé : " notre déambulation de couple s'étirait, une heure durant, puis nous rejoignons le port." NMP., p.351; durant cette promenade, la narratrice se souvient de l'un de ses parents qui était le demi-frère de la grand-mère maternelle à la cité, autrement dit l'oncle maternel de sa mère, elle l'appelle ainsi : "Khali Tadder" NMP., p.352; La jeune fille exprime alors sa nostalgie et son désir fort de retourner à ce passé lointain.

\section{Le huitième intertitre "8. MOUNIRA RÉAPPARUE":}

Dans cette section, la narratrice est alors admise à l'université, et elle raconte sa rencontre imprévue avec Mounira, l'une de ses

(Texte et paratexte dans ...) Ibrahim Abouelmaati Ibrahim Elmorsy 
condisciples au collège, à la sortie de la bibliothèque universitaire où elle doit "traduire un texte de Lucrèce que le professeur de latin nous avait demandé." NMP., p.361; la narratrice est très choquée de la curiosité et l'intrusion de Mounira dans ses affaires. Celle-ci a aussi demandé de nouvelles concernant la relation de la narratrice avec Tarik : "elle insiste pour savoir comment je fais pour rencontrer mon "amoureux""'. NMP., p.363 La narratrice avait un rendez-vous avec Tarik, bien sûr à l'insu de ses parents ; mais l'intrigante Mounira a voulu fortement les rejoindre et la jeune fille a accepté.

\section{Le neuvième intertitre "9. NOUS... NOUS !":}

A la brasserie Victor Hugo, la narratrice raconte sa rencontre avec Tarik en donnant une description de Mounira et aussi de son acte : "habillée de couleurs vives, le visage fardé, les paupières soulignées d'un trait bleu. Elle répond brièvement à mon salut et s'assoit d'autorité auprès de Tarik, qui s'est courtoisement levé." NMP., p.368; la narratrice raconte également comment cette fausse amie a monopolisé la conversation, elle décrit aussi son comportement indécent avec Tarik en oubliant sa présence : "Mounira se penche vers Tarik (...) La tête tournée vers Tarik (...) s'adresse à nouveau ostensiblement au seul Tarik." NMP., p.369; alors la narratrice vexée a pris la décision de mettre fin à cette rencontre, elle s'adresse à Mounira en disant :" Comme je te l'ai dit hier, nous avons maintenant un programme à nous, on va te dire au revoir." NMP., p.370; Mais devant cette initiative de la narratrice, Tarik lui exprime son mécontentement, sa désapprobation sur son comportement en lui demandant en forme d'ordre : " tu vas retourner vers ta copine, la ramener jusqu'à nous, et ..." NMP., p.374 Alors, la narratrice fait la comparaison entre Tarik et son père : "tous deux réunis me donneraient un tel ordre..." NMP., p.374; et après, elle a qualifié Tarik par:

(Texte et paratexte dans ...) Ibrahim Abouelmaati Ibrahim Elmorsy 
"l'homme, le même-pas-fiancé" NMP., p.375 elle n'accepte pas les ordres, elle a déjà décidé de jouir de sa liberté : "oublions l'homme qui divague! Marche loin, devant toi, toujours devant... jusqu'à la mer!" NMP., p.375

\section{Le dixième intertitre "10. DANS LE NOIR VESTIBULE...":}

La narratrice se sent profondément perdue en exprimant son malaise et son état d'âme tourmenté et brisé ; elle se pose des questions qui expriment son désespoir, citons à titre d'exemple: "Comment ai-je continué à vivre malgré cette opacité nouée en moi, n'ayant certes causé du tort qu'à moi-même, à la part féminine, dirais-je, de mon cour ?" NMP., p.378

En s'éloignant de Mounira, la narratrice et Tarik commencent à se discuter fortement tout au long d'une rue étroite en pénétrant "dans un hall d'immeuble (...) une fois nous deux face à face dans le noir" NMP., p.379 La narratrice choquée ressent dès lors qu'elle est sans domicile : "Dès la première minute de face à face, je sais, je sens en effet que je n'ai plus de lieu! Je n'aurai même plus la maison de mon père!" NMP., p.380

Après avoir vu la dureté et l'amour du pouvoir dans le visage et la parole de Tarik, elle se met à réfléchir au risque et au danger dans lesquels elle va vivre si son père est au courant de cette relation, la narratrice dit :" Si mon père l'apprend, je me tue." NMP., p.385

La narratrice ne cesse de répéter cette phrase :" Si mon père le sait, je me tue." NMP., ppp.390-391-392 ; la narratrice est donc dévorée par le remords et l'inquiétude.

\section{Le onzième intertitre "11. CE MATIN-LÀ":}

Après une tentative de suicide manquée, la narratrice a trouvé la solution voire le remède qui peut la faire sortir de cet état d'âme déchiré et de cette solitude : c'est au Coran qu'elle recherche refuge ; elle dit : "Un jour, ouvrant un Coran à une page au

(Texte et paratexte dans ...) Ibrahim Abouelmaati Ibrahim Elmorsy 
hasard, car j'étais en complet désarroi (...) Je quêtais intérieurement une "lettre", un verset, une parole à moi destinés : en somme un signal! "Nul ne peut porter la charge de l'autre" disait le verset dans la sourate de l'Étoile au moment où je mendiais, en silence, de l'aide." NMP., p.402; la narratrice explique l'impact de la densité de ce verset sur elle:" Je la saisis comme une lueur pour sortir de l'obscurité." NMP., p.403; un autre verset de la même sourate dit : "L'étoile, lorsqu'elle tombe..." je le relis parfois: il me rappelle, à moi redevenue sereine, mon tout premier désarroi." NMP., p.403

La narratrice découvre plus tard que son amour n'était pas pour ce jeune homme inconnu ; mais pour ce qu'il possède d'habileté à maitriser sa langue maternelle ; elle reconnait finalement qu'elle s'est sentie amoureuse :" de la langue perdue, réanimée dans ce visage de jeune homme qui la maîtrisait, lui, cette langue d'autrefois." NMP., p.408

La narratrice insiste sur la répétition de cette phrase, exprimée de manières différentes, qui souligne à quel point cette jeune fille est obsédée par la peur et aussi le respect de son père absent: "Si mon père l'apprend, je me tue! (...) si mon père me convoque à son tribunal, je n'apparaîtrai pas ! (...) Si je suis convoquée au tribunal du père...je me tue! (...) Si mon père...je me tue! Si mon père... je me tue ! (...) Si mon père le sait... je me tue... tue !" NMP., pp.409-410 ; mais la narratrice, après cette phrase tant répétée, se souvient de sa croyance religieuse en disant : "Le suicide est interdit en islam!" NMP., p.411

De son point de vue, ce sont le père et Tarik qui la poussent à la tentative du suicide, et qui représentent la raison essentielle de sa perte et son enfermement; la narratrice décrit les deux en disant: "le père, victime de son ignorance rigoriste et des préjugés de son groupe, et surtout ledit "fiancé", faux chevalier en proie aux

(Texte et paratexte dans ...) Ibrahim Abouelmaati Ibrahim Elmorsy 
ombres de sorcières ou d'envieuses, femmes anges et putains qui l'avaient entouré, adulé, annihilé." NMP., p.415

La narratrice indique finalement que son refus de cette vie où prédominent les hommes et sa décision d'aller poursuivre ses études universitaires à l'étranger datent de 1953; autrement dit avant le déclenchement de la Guerre de l'Algérie contre la colonisation française; ici la narratrice fait allusion à cet événement majeur dans l'Histoire de son pays, cette guerre qui représente aussi un refus de tout genre d'autorité, soit colonisatrice, soit masculine :" Ce jour d'octobre 1953 précéda de douze mois l'explosion d'un pays, de toute la terre dite Algérie." NMP., p.415

\section{ÉPILOGUES :}

Après la troisième partie du roman, on trouve un autre élément paratextuel, c'est l'épilogue qui contient ici trois sections à la fois numérotées et intitulées. L'épilogue : "désigne, en général une partie finale ajoutée, comme de surcroit, à un discours, à un ouvrage, en lui-même complet." (29)

\section{Le premier intertitre "1. LE SILENCE OU LES ANNÉES- TOMBEAUX":}

A travers cet intertitre "Le silence ou les années-tombeaux", Assia Djebar insiste sur la situation misérable de toute femme algérienne. Comme la vie nécessite la parole et le silence à la fois, l'auteure décrit la période de vie silencieuse de la femme comme si celle-ci est enterrée dans la tombe. La romancière indique alors le silence dans lequel vit toute femme, elle n'a pas le droit de s'exprimer, de donner son point de vue; c'est pour cela elle garde toujours le silence. L'écrivaine a voulu dire que la vie en silence est tout près de la mort; en vain, elle cherche toujours le dialogue

(Texte et paratexte dans ...) Ibrahim Abouelmaati Ibrahim Elmorsy 
dans son milieu familial ancré dans les traditions du passé lointain.

La narratrice-auteure commence cette section en posant des questions qui résument peut-être la forme générale du roman : "Ce récit est-il le roman d'un amour crevé ? Ou la romance à peine agitée d'une jeune fille, j'allais dire "rangée"- simplement non libérée- du sud de quelque méditerranée ? L'esquisse d'une ouverture, prologue à une plus vaste autobiographie ?" NMP., p.419; à travers ces trois questions sans réponses, elle pousse son lecteur à bien comprendre le contenu du roman. La narratrice exprime alors sa souffrance comme une jeune fille privée de toute liberté au-dedans de son milieu familial, malgré la métamorphose de son père à la fin. L'absence de liberté l'a conduite à cacher plusieurs choses et même à mentir. Elle n'a pas réussi à trouver le bonheur. Assia Djebar affirme que ce roman ressemble plus ou moins à une grande partie de sa vie privée. Elle s'avoue : "moi, ici personnage et auteur à la fois" NMP., p.419

Malgré son enfermement, la narratrice parle de sa maison paternelle en défendant le père qui assume ses responsabilités comme le protecteur de sa famille dont les membres sont la femme, la fille, la mère et les sœurs; il "redevient malgré lui ou sans le savoir "gardien de gynécée." NMP., p.421; Anne Marie Miraglia apprécie que : "Finalement, Tahar est examiné sous le regard de sa fille quinquagénaire, désormais orpheline, qui brosse le portrait d'un homme austère, culturellement hybride." (30)

La narratrice souligne donc qu'elle n'a pas d'autre choix, elle était obligée de suivre cette route sans fin, c'est dans la lecture qu'elle a essayé d'échapper à tout ce qui l'entoure en gardant le silence, en oubliant sa vie parentale : "Je me suis engloutie à force de m'être

(Texte et paratexte dans ...) Ibrahim Abouelmaati Ibrahim Elmorsy 
tue (...) Se taire devant soi-même : ce fut le plus grave." NMP., p.426

Assia Djebar termine aussi cette section avec une question concernant son enfance passée et le foyer paternel :" est-ce là-bas que je cherche, moi, inlassable, où se trouve la petite, l'obscure maison de mon père?" NMP., p.428.

\section{Le deuxième intertitre "2. LA JEUNE FILLE SAUVAGE":}

La narratrice se souvient ici de son accident du tramway et comment elle était "rigide, les yeux secs" NMP., p.429; mais elle souhaite une autre fin de son récit: "je regrette que le sort, ou la chance, ou le hasard qui, par essence, est neutre, oui, vraiment, je souffre du fait que ce conducteur du tramway (...) n'ait pas laissé sa machine lancée en trombe poursuivre son élan! L'on aurait retiré mon corps en morceaux..." NMP., p.431

La narratrice a voulu exprimer son désespoir en ce moment-là, et cela revient à sa vision énigmatique et aussi décevante pour le futur, elle pose la question en donnant la réponse :" Dès lors, pourquoi ce regret? Pour l'inutilité des décennies à venir, pour le gel intérieur qui me maintint." NMP., p.432

La narratrice affirme enfin sa déception de trouver un lieu dans la maison paternelle et finit cette section en posant une question concernant le sort de toutes les femmes de sa génération: "Pourquoi, mais pourquoi faut-il que je me retrouve, moi et les autres, "nulle part dans la maison de mon père"?" NMP., p.433

\section{Le troisième intertitre "3. INVENTER LE VERTIGE":}

La narratrice, voire l'écrivaine, aborde ici son itinéraire dans l'univers de l'écriture qui a duré plus de cinquante ans, une période très difficile dans sa vie : "Ecrire tantôt avec des larmes (...), tantôt pour capter un rythme, depuis ce jour d'automne 1953 jusqu'en 2007, plus d'un demi-siècle après. "NMP., p.435

(Texte et paratexte dans ...) Ibrahim Abouelmaati Ibrahim Elmorsy 
Assia Djebar traite encore une fois l'insurrection de son peuple algérien contre la présence coloniale, les deux camps se sont affrontés: "en cette nuit du $1^{\text {er }}$ novembre 1954" NMP., p.437; et la mort a gagné des hommes, des femmes et des enfants, tandis que l'auteure se sent du malaise, elle ne fait qu'écrire, elle s'adresse à soi-même : " alors que toi, tu n'es désormais de nulle part (...) te voici donc écrire" NMP., p.437; la narratrice auteure souffre toujours, la solitude l'entoure et la domine ; mais elle dit: "Je n'ai pas peur, j'ai seulement le vertige!"

A la fin de l'épilogue, Assia Djebar fait allusion aux lieux et au temps où elle séjourne pour écrire, inventer et remémorer, loin de sa maison paternelle :

"New York: novembre 2005-février 2006

New York et Paris: novembre 2006-mai 2007" NMP., p.441

POSTFACE "SILENCE SUR SOIE" OU L'ÉCRITURE EN FUITE":

Vient à la fin du roman le dernier élément paratextuel, c'est la postface ; Genette détermine sa fonction : "Par son emplacement et son type de discours, la postface ne peut espérer exercer qu'une fonction curative, ou corrective."

Assia Djebar a choisi pour la postface un intertitre "Silence sur soie" ou l'écriture en fuite" et dans la première ligne, elle dit : "J'aurais pu intituler ce texte Silence sur soie" NMP., p.445; ainsi, elle a voulu montrer que sa vie était pleine de silence malgré que son silence était soyeuse. Elle a choisi également plusieurs dénominations de cet ouvrage littéraire, citées toutes dans la postface ; citons par exemple: "écrire sur soi"p.445, "auto-analyse rétrospective"p.445, "confession"p.445, "écrit sur soi"p.446, "un livre"p.451, "entreprise"p.451. C'est à travers l'écriture que l'écrivaine a triomphé sur son silence et a pu

(Texte et paratexte dans ...) Ibrahim Abouelmaati Ibrahim Elmorsy 
s'exprimer. Elle déclare que "Cette confession (...) peut m'inciter pourtant à battre ma coulpe, tout en flattant peut-être ma vanité d'écrivain." NMP., p.445

Dans cette conclusion brève d'un roman volumineux, la narratrice auteure exprime alors sa longue carrière comme écrivaine en avouant que son texte n'était : "ni désir compulsif de la mise à nu, ni hantise de l'autobiographie- ce succédané "laïcisé" de la confession en littérature d'occident." NMP., p.446; elle s'avoue donc que son ouvrage littéraire était basé sur une certaine période de sa vie :" un premier cercle de souvenirs - enfance, puis adolescence culminant dans un soudain "acte gratuit" lors de ma dix-septième année." NMP., p.446 Ainsi, il est clair qu'Assia Djebar emprunte les titres des parties et les intertitres aux épisodes très proches de sa vie personnelle.

À la dernière page de la postface, l'auteure donne à son lecteur sa signature en soulignant la date et le lieu de l'écriture de son ouvrage: "A. D. -2006-2007-New York-Paris." NMP., p.451

Hafid Gafaïti estime que :" C'est par l'écriture qu'Assia Djebar entre dans l'Histoire et par elle qu'elle se fonde comme sujet. Et c'est à partir de l'écriture conçue comme conjonction entre l'individuel et le collectif qu'elle se sentira investie en tant qu'Algérienne pour parcourir l'Histoire de son pays et en tant que femme pour la réécrire d'un point de vue féminin, avec et pour les autres femmes." ${ }^{(32)}$

D'après Genette, c'est dans la postface " où l'auteur pourrait épiloguer en toute connaissance de cause de part et d'autre : "Vous en savez maintenant autant que moi, alors causons." (33)

\section{La quatrième de couverture :}

Autre élément paratextuel qui attire également le lecteur virtuel du livre : c'est la page 4 de couverture qui, d'après Gérard

(Texte et paratexte dans ...) Ibrahim Abouelmaati Ibrahim Elmorsy 
Genette, forme pour le lecteur un petit résumé sur le contenu de l'ouvrage présenté. C'est la meilleure façon de se faire une idée sur un roman que je n'ai pas encore lu. Selon Genette, cet élément contient plusieurs notices qu'on trouve dans "Nulle part dans la maison de mon père" : rappel de la maison d'édition et du titre de l'ouvrage, une notice biographique et/ou bibliographique concernant l'ouvrage et l'auteure, un manifeste de collection, la référence de l'illustration de couverture, le prix de vente, le numéro ISBN, le code-barre magnétique. Toutes ces notices paratextuelles figurées sur la page 4 de couverture ont pour objectif de donner au lecteur toutes les informations nécessaires, groupées sur une seule page, qui le poussent à considérer la valeur et l'intérêt du livre. Parmi ces éléments, on analyse la notice biographique qui est présentée comme ça :

" Dans une petite ville du littoral algérien, une fillette grandit entre son père, seul instituteur indigène de l'école, et sa mère, si belle et si jeune. Livrée à la houle des souvenirs, Assia Djebar évoque sa formation et ses figures tutélaires au premier rang desquelles son père, l'austère Tahar qui, malgré ses idéaux démocratiques, reste attaché à une rigueur musulmane qu'il entend transmettre à sa fille. C'est par les livres qu'elle découvre le monde - bientôt viendra l'écriture.

Pour la première fois dans son œuvre, Assia Djebar compose un roman autobiographique qui éclaire son identité de femme et d'écrivain : on y découvre une enfant puis une jeune fille avide de liberté, riche d'une tradition en héritage et d'un savoir qu'elle conquiert brillamment, déchirée entre l'Algérie et la France. Au-delà du récit intime tout en pudeur et en émotion, elle rend hommage à un passé arabo-berbère, à un pays, à un père, comme pour renouer des liens dont elle a naguère dû s'affranchir pour devenir ellemême.

Née en Algérie en 1936, Assia Djebar a publié son premier roman, La Soif, en 1957. Auteur d'une cuvre importante traduite dans le monde entier, membre de l'Académie royale de Belgique et docteur honoris causa de plusieurs universités prestigieuses, Assia Djebar est entrée à l'Académie française en 2006."

Ainsi on trouve, dans le premier paragraphe, un petit résumé du roman; plus de quatre cents pages se présentent en quelques

(Texte et paratexte dans ...) Ibrahim Abouelmaati Ibrahim Elmorsy 
lignes, l'éditeur met en avant l'histoire racontée dans le roman. Et le deuxième paragraphe souligne non seulement l'indication générique de cet ouvrage mais aussi la vraisemblance de ses événements tous reliés à l'auteure, c'est un roman autobiographique. Et enfin et en italique, on trouve une biographie brève concernant Assia Djebar, dans laquelle l'éditeur, à côté des faits factuels (lieu et date de naissance), présente tout ce qui distingue l'écrivaine des autres, tout ce qui la valorise comme son admission à l'Académie française.

Ainsi, on constate la relation étroite entre le texte "le contenu du roman" et le paratexte "éléments qui entourent le texte"; il est remarquable que ces éléments paratextuels contribuent fortement à une meilleure compréhension du roman. Ces indices aident le lecteur à bien identifier l'auteure et son œuvre. Assia Djebar présente toujours une œuvre touffue d'un bon nombre des données paratextuelles, plus ou moins attachées au texte lui-même, qui se lient ensemble C'est pourquoi nous avons choisi l'étude de ces éléments en dévoilant leur relation avec le texte.

Grâce aux indices paratextuels, le lecteur peut entrer immédiatement en contact avec l'œuvre, en obtenant des renseignements, bien présentés de la part de l'écrivain, qui le guident à suivre le chemin dessiné par l'auteur.

Entre le texte et le paratexte s'établit une relation étroite, c'est pour cette raison qu'on met en rapport les données paratextuelles avec les événements narrés par l'auteure. Généralement, l'écriture d'Assia Djebar touche une grande partie de sa vie; il est remarquable que son originalité réside dans le choix des thèmes abordés, dans son désir de révolte contre tout ce qui est traditionnel. Nulle part dans la maison de mon père a bien exprimé une certaine vision de la société algérienne bien ancrée dans la mémoire de cette écrivaine qui s'avoue: "Je voudrais me

(Texte et paratexte dans ...) Ibrahim Abouelmaati Ibrahim Elmorsy 
présenter devant vous comme simplement une femme-écrivain, issue d'un pays, l'Algérie tumultueuse et encore déchirée. J'ai été élevée dans une foi musulmane, celle de mes aïeux depuis des générations, qui m'a façonnée affectivement et spirituellement, mais à laquelle, je l'avoue, je me confronte, à cause de ses interdits dont je ne me délie pas encore tout à fait." ${ }^{\prime(34)}$

Ainsi, Assia Djebar a terminé son parcours romanesque par un ouvrage romanesque distingué, bien différent de ses précédents, qui touche sa vie personnelle et aussi celle de toute femme algérienne. Nulle part dans la maison de mon père, à partir de son titre et de sa volume, attire l'attention du lecteur. C'est à travers le titre que naît l'idée de notre recherche qui regroupe le texte et la paratexte ensemble. 


\section{Notes :}

( $\left.{ }^{1}\right)$ Agar-Mendousse (Trudy), Violence et créativité de l'écriture algérienne au féminin, éd. L'Harmattan, Paris, 2006, p19

(2) Genette (Gérard), Seuils, éd. Seuil, Paris, 1987, p.7

(3) Cf., Genette (Gérard), Seuils, Op. Cit. , pp.10-11

(•) Terme spécifique à Gérard Genette signifiant "la première page de couverture".

(") "J'appelle péritexte éditorial" toute cette zone du péritexte qui se trouve sous la responsabilité directe et principale (mais non exclusive) de l'éditeur, ou peut-être, plus abstraitement mais plus exactement, de l'édition. "Genette, Seuils, p.20"

$\left({ }^{4}\right)$ Genette (Gérard), Seuils, Op. Cit., p.40

$\left({ }^{5}\right)$ www.Leblog d'Assia Djebar

$\left({ }^{6}\right)$ Heller (Michael), Assia Djebar, Une conversation avec Michael Heller, in Cahiers d'Études Maghrébines, $\mathrm{N}^{\circ}$ 14, Spécial Assia Djebar, 22 octobre 2000, p. 56.

( ${ }^{7}$ ) https://madame.lefigaro.fr/prenoms/prenom/fille/assia

$\left({ }^{8}\right)$ Chatti (Mounira), Assia Djebar, la voix des autres, in Nouvelles Études Francophones, Volume 30, Numéro 1, Printemps 2015, p.1

$\left.{ }^{9}\right) \quad$ https://www.franceculture.fr/emissions/une-vie-une-oeuvre/assia-djebarune-vie-entre-deux-rives-1936-2015

$\left({ }^{10}\right)$ Grivel (Charles), Production de l'intérêt romanesque, La Haye : Mouton, Paris, 1973, p. 173.

( ${ }^{11}$ ) Hoek (Léo. H.), La Marque du titre, La Haye, Mouton, Paris, 1981, p. 1.

$\left({ }^{12}\right)$ Bourkhis (Ridha), La rhétorique de la passion dans le texte francophone, Mélanges offerts à Jean Déjeux, éd. L'Harmattan, Paris, 2010, p.68

$\left({ }^{13}\right)$ Jouve (Vincent), La poétique du roman, éd. SEDES, Paris, 1997, p. 13.

$\left({ }^{14}\right)$ Cf., Genette (Gérard), Seuils, Op. Cit., p. 75

$\left({ }^{15}\right)$ Cf., Hoek (Léo. H.), La Marque du titre, Op. Cit., p. 189

$\left({ }^{16}\right)$ Genette (Gérard), Seuils, Op. Cit., p. 80

(") Voir les connotations de ces intertitres en détail à partir de la page 15 jusqu'à la page 39 .

$\left({ }^{17}\right)$ (Miraglia) A. M., Cet amour de père, gardien du gynécée, in Études françaises, 52 (1), 2016, p.2 (disponible sur :

https://doi.org/10.7202/1035540ar)

$\left({ }^{18}\right)$ https://www.cnrtl.fr/etymologie/part ( Centre National de Ressources Textuelles et Lexicales)

(Texte et paratexte dans ...) Ibrahim Abouelmaati Ibrahim Elmorsy 
$\left({ }^{19}\right)$ (Bourkhis) Ridha, La rhétorique de la passion dans le texte francophone, Mélanges offerts à Jean Déjeux, Op. Cit., p. 69

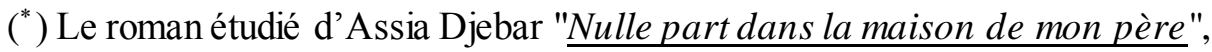
cité fréquemment tout au long de l'article, sera identifié avec cet acronyme : "NMP."

$\left.{ }^{20}\right)$ (Bourkhis) Ridha, La rhétorique de la passion dans le texte francophone, Mélanges offerts à Jean Déjeux, Op. Cit., p. 69

$\left({ }^{21}\right)$ Genette (Gérard), Seuils, Op. Cit., p.89

${ }^{22}$ ) Cf., Genette (Gérard), Seuils, Op. Cit., p.95

$\left({ }^{23}\right)$ Chatti (Mounira), Mardi 24 avril 2012, http://Assia Djebar. Club de lecture. blogs pot

$\left({ }^{24}\right) \mathrm{http} / / / \mathrm{www}$.univ-paris3.fr/gayatri-spivak-dans-le-siecle-politiques-delecture-et-ecriture-pour-refonder-une-imagination-critique-autour-destravaux-de-gayatri-spivak--474485.kjsp

$\left({ }^{25}\right)$ Genette (Gérard), Seuils, Op. Cit., pp.148-149

$\left({ }^{26}\right)$ Ibid., p.271

$\left({ }^{27}\right)$ Cf., Genette (Gérard), Seuils, Op. Cit., p. 274

(*) Terme spécifique à Gérard Genette (Seuils, p.280).

(*) Hector Malot est un romancier français, né en 1830, mort en 1907, et devenu très célèbre lors de la parution de son roman "Sans Famille" en 1878.

(28) Ali-Benali (Zineb), Assia Djebar, Ecrire, entre voix et corps, Histoire de soi, histoire des siens, éd. Centre culturel du livre, Casablanca, 2019, p.23

(*) "Les Cloches de Corneville" est une opérette en trois actes, composée par Robert Planquette et considérée comme l'un des ouvrages lyriques français les plus populaires; opéra-comique avec lequel Planquette a connu une immense notoriété. (Cf.,https://www.epmmusique.fr/fr/cd-operettes-operas/2654-les-cloches-decorneville-planquette.html)

$\left.{ }^{(29}\right)$ https://www.espacefrancais.com/le-prologue-et-lepilogue/

$\left.{ }^{30}\right)$ Miraglia (Anne Marie), Cet amour de père, gardien du gynécée, in Études françaises, 52 (1), 2016, p.40. disponible sur (https://doi.org/10.7202/1035540ar)

$\left({ }^{31}\right)$ Genette (Gérard), Seuils, Op. Cit., p. 220

${ }^{32}$ ) Gafaiti (Hafid), Les femmes dans le roman algérien, éd. L'Harmattan, Paris, 1996, p.221

$\left({ }^{33}\right)$ Genette (Gérard), Seuils, Op. Cit., p.219

$\left({ }^{34}\right)$ Extrait du discours prononcé à Francfort par Assia Djebar pour la remise du Prix de la Paix en octobre 2000, disponible sur : http://remue.net/Idiomede-l-exil-et-langue-de-1-irreductibilite-par-Assia-Diebar

(Texte et paratexte dans ...) Ibrahim Abouelmaati Ibrahim Elmorsy 


\section{Bibliographie:}

\section{Le roman étudié :}

- Nulle part dans la maison de mon père, éd. Babel, 2007, 451 pages.

Ouvrages consacrés partiellement ou totalement à Assia Djebar ou à la littérature maghrébine d'expression française :

- Agar-Mendousse (Trudy), Violence et créativité de l'écriture algérienne au féminin, éd. L'Harmattan, Paris, 2006

- Bourkhis (Ridha), La rhétorique de la passion dans le texte francophone, Mélanges offerts à Jean Déjeux, éd. L'Harmattan, Paris, 2010

- Ali-Benali (Zineb), Assia Djebar, Ecrire, entre voix et corps, Histoire de soi, histoire des siens, éd. Centre culturel du livre, Casablanca, 2019

- Gafaiti (Hafid), Les femmes dans le roman algérien, éd. Le Harmattan, Paris, 1996

\section{Ouvrages généraux :}

- Genette (Gérard), Seuils, éd. Seuil, Paris, 1987

- Grivel (Charles), Production de l'intérêt romanesque, La Haye : Mouton, Paris, 1973

- Hoek (Léo.H.), La Marque du titre, La Haye, Mouton, Paris, 1981

- Jouve (Vincent), La poétique du roman, éd. SEDES, Paris, 1997

\section{Thèses consultées :}

- Labontu-Astter (Diana), L'image du corps féminin dans l'œuvre d'Assia Djebar, thèse de doctorat, Université de Grenoble, 2012.

- Bengaffour (Nawal), L'écriture de l'errance dans les ceuvres d'Assia Djebar, Université d'Oran, 2009-2010

- Mokhtari (Fatima Zohra), Récit de filiation ou écriture du père chez Maissa Bey, Malika Mokeddem et Assia Djebar, thèse de

(Texte et paratexte dans ...) Ibrahim Abouelmaati Ibrahim Elmorsy 
doctorat, Faculté des Langues étrangères, Université d'Oran 2, 2018-2019

- Van Deventer (Rachel), L'Agentivité et la naissance de la femme-sujet dans la littérature algérienne contemporaine, Faculté des Études supérieures, Université d'Ottawa, Canada, 2010

\section{Articles et périodiques:}

- Miraglia (Anne Marie), Cet amour de père, gardien du gynécée, In Etudes françaises, Volume no 52, Numéro (1), 2016.

- Chatti (Mounira), Assia Djebar, la voix des autres, in Nouvelles Études Francophones, Volume 30, Numéro 1, Printemps 2015.

- Heller (Michael), Assia Djebar, Une conversation avec Michael Heller, in Cahiers d'Études Maghrébines, $\mathrm{N}^{\circ} 14$, Spécial Assia Djebar, 22 octobre 2000.

\section{Sitobiographie :}

- https://www.cnrtl.fr/etymologie/part

- https://www. franceculture.fr/emissions/une-vie- uneoeuvre/assia-djebar-une-vie-entre-deux-rives-1936-2015

- http://remue.net/Idiome-de-l-exil-et-langue-de-l-irreductibilitepar-Assia-Djebar

- ( ) https://www.espacefrancais.com/le-prologue-et-lepilogue/

- https://digitalcommons.lsu.edu/grad school_dissertations/3153

- http://journals.openedition.org/multilinguales/835

- http:/journals.openedition.org/multilinguales/860

- www.Leblog d'Assia Djebar

- https://madame.lefigaro.fr/prenoms/prenom/fille/assia

- http://www.univ-paris3.fr/gayatri-spivak-dans-le-sieclepolitiques-de-lecture-et-ecriture-pour-refonder-une-imaginationcritique-autour-des-travaux-de-gayatri-spivak--474485.kjsp

- https://www.epmmusique.fr/fr/cd-operettes-operas/2654-lescloches-de-corneville-planquette.html

(Texte et paratexte dans ...) Ibrahim Abouelmaati Ibrahim Elmorsy 
- http://assiadjebarclubdelecture.blogspot.com/

- http://Assia Djebar. Club de lecture. blogs pot

- http://africultures.com/personnes/?no=3798

- http://www.laroseraiedescultures. fr/edition2012/mb-hommagea-assia-djebar.html\#b

- https://www.livrescq.com/livrescq/amel-chaouati-fondatrice-ducercle-des-amis-dassia-djebar/

- http://assiadjebar.canalblog.com/

- https://www.limag.com/new/index.php?inc=iframe\&file=Textes /Herzberger/DjebarPrix.htm

- https://www.limag.com/new/index.php?inc=iframe\&file=Textes /Perfler/Djebar.htm

- https://www.limag.com/new/index.php?inc=iframe\&file=Textes /Regaieg/RegaiegDjebarNuits.htm

- https://www.limag.com/new/index.php?inc=dspart\&art=000361 11 


\section{ملخص}

آسيا جبار هي واحدة من أبرز الكتاب الجزائريين الناطقين بالفرنسية. انتخبت آسيا جبار في الأكاديمية الفرنسية عام ه . . F ، وهي مؤلفة لعدد كبير من الروايات التي تتتاول جزءًا مهمًا جدًا من تاريخ بلدها ، وقبل كل شيء الدفاع عن حرية المرأة العربية. ثُرجمت أعمالها الروائية إلى العديد من اللغات الأجنبية.

من بين أعمالها الروائية اخترنا روايتها الأخيرة" لا مكان في منزل أبي "والتي

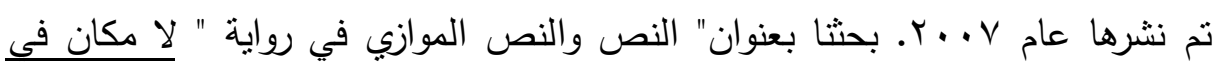
منزل أبى "لأسيا جبار •"إن هذه الرواية تحتوي علي مجموعة كبيرة من العناصر الموازية للنص التي أدهشتا ودفعتتا لاختيار هذا الموضوع. قمنا بتحليل كل عنصر موازي للنص وعلاقته بالنص نفسه والكاتبة. من بين تلك العناصر التي قمنا بتتاولها نذكر علي سبيل المثال : العنوان ,اسم المؤلف، دار النشر، الاهداء، العناويين، الخاتمة ...إلخ

قدمت لنا آسيا جبار عملا مختلفا تماما عن الأخرين ببنيته الفريدة ، وقد نجحت في إبراز كل عنصر من عناصر النص الموازي في الرواية بطريقة منطقية. يمثل هذا العمل علامة مميزة في مسيرتها الروائية ، فقد برعت في الوصول إلى قارئها من عنوان الرواية الذي يمثل العنصر الموازي للنص الأكثر تميزا والأكثر جذبا لانتباه كل قارئ. 